\title{
RESPUESTA FISIOLÓGICA DE Euglena gracilis AL ESTRÉS POR COBRE
}

\author{
David Cervantes García, Daniel González-Mendoza*, Lourdes Cervantes-Díaz, Adriana Morales Trejo y Onecimo \\ Grimaldo Juárez
}

Instituto de Ciencias Agrícolas, Universidad Autónoma de Baja California, Carretera a Delta s/n, Código Postal 21705, Ejido

Nuevo León - Baja California, México

Roberto Zamora-Bustillos

Instituto Tecnológico de Conkal, km 16.3, Antigua Carretera Mérida-Motul, C. P. 97345 Conkal - Yucatán, México

Recebido em 1/1/11; aceito em 21/2/11; publicado na web em 15/4/11

\begin{abstract}
PHYSIOLOGICAL RESPONSES OF Euglena gracilis TO COPPER STRESS. The objective of this study was to evaluate the toxic effect of $\mathrm{Cu}^{2+}$ in the physiological development of E. gracilis. The results showed that $E$. gracilis had an effect on the dose-dependent growth to the concentration of metal. The exposure of E. gracilis metal at doses of 0.8 and $1.6 \mathrm{mM}$ of $\mathrm{Cu}^{2+}$ showed a significant negative effect on the stability of DNA and photosynthetic pigments involved in capturing light in the antenna complex after $144 \mathrm{~h}$ of exposure to the metal.
\end{abstract}

Keywords: Euglena gracilis; copper; photosynthetic pigments.

\section{INTRODUCCIÓN}

Durante, las últimas décadas, como resultado del desarrollo de asentamientos poblacionales y el impulso de diferentes actividades industriales se ha favorecido una mayor persistencia y bio-disponibilidad de compuestos químicos como los metales pesados en diferentes ecosistemas acuáticos. ${ }^{1}$

Los elementos potencialmente tóxicos (EPTs) pueden ser esenciales o no esenciales para los seres vivos, pero todos ellos en exceso representan un riesgo latente para la salud de las especies que habitan estos ecosistemas entre ellas al ser humano. Dentro de los EPTs que tienen gran relevancia en la agricultura es el Cobre $\left(\mathrm{Cu}^{+2}\right)$, el cual es requerido en el metabolismo y procesos celulares de las plantas, pero en cantidades elevadas puede generar serios problemas en la salud de los sistemas acuáticos. ${ }^{2}$

Lo anterior ha generado la necesidad de desarrollar tecnologías para la remediación de ambientes acuáticos afectados por metales. En este sentido, el uso de microorganismos tolerantes con capacidad de almacenar metales ha demostrado ser una biotecnología con ventajas sobre los métodos físicos-químicos, ya que ofrece un menor impacto ambiental y menor costo económico. ${ }^{3}$ En este sentido el protista acuático de vida libre Euglena gracilis representa un modelo biológico idóneo para ser empleado en procesos de biorremediación. Este microorganismo forma parte del plancton de aguas dulces y puede ser cultivado de manera eficiente en condiciones controladas de laboratorio y con diferentes fuentes de carbono. Además presenta propiedades metabólicas y genéticas que le permite desarrollarse en presencia de altas concentraciones de metales esenciales y no esenciales a diferentes valores de $\mathrm{pH}$ y bajo un régimen heterotrófico o fotosintético. ${ }^{4}$

Estas propiedades hacen que Euglena gracilis pueda ser considerada como un organismo con potencial biotecnológico en la biorremediación de sistemas acuáticos impactados por EPTs. En la actualidad existen diversos trabajos sobre el estudio del efecto de metales en este

*e-mail: daniasaf@gmail.com organismo siendo el cadmio el metal mas estudiado. ${ }^{5}$ Sin embargo; el efecto toxico del cobre en la fisiología de E. gracilis ha sido poco abordado, por lo tanto, el objetivo del presente estudio fue evaluar el efecto toxico del $\mathrm{Cu}$ en el desarrollo fisiológico de E. gracilis.

\section{PARTE EXPERIMENTAL}

Se realizaron experimentos empleando cultivos axénicos de Euglena gracilis cepa Z (generosamente donada por el Dr. T. Ishikawa). El experimento consistió en realizar el crecimiento del microorganismo en un medio de cultivo mínimo con la siguiente formulación: acetato de sodio ( $1 \mathrm{~g} / \mathrm{L})$, extracto de carne (1 g/L), Triptona ( $2 \mathrm{~g} / \mathrm{L})$, extracto de levadura $(2 \mathrm{~g} / \mathrm{L})$ y $\mathrm{CaCl}_{2} \mathrm{H}_{2} \mathrm{O}_{2}(10 \mathrm{mg})$. Los experimentos se realizaron inoculando $1 \times 10^{6}$ de células obtenidas de la fase exponencial (6 días de crecimiento) en matraces de $50 \mathrm{~mL}$ con $0,0.05,0.2,0.4,0.6,0.8 \mathrm{y}$ $1.6 \mathrm{mM} \mathrm{de} \mathrm{CuCl}_{2}$. Los matraces se mantuvieron bajo luz blanca fría continua con una intensidad lumínica de $90 \mu \mathrm{mol} \mathrm{m}{ }^{-2} \mathrm{~s}^{-1} \pm 10 \mu \mathrm{mol} \mathrm{m}{ }^{-2}$ $\mathrm{s}^{-1}$, a una temperatura de $24 \pm 2{ }^{\circ} \mathrm{C}$ y con agitación manual dos veces al día. La densidad celular (N) se determinó cada 24 h por conteo directo en microscopio utilizando cámara de Neubauer durante 7 días y se determinó la tasa de crecimiento $\mathrm{k}$ en número de divisiones celulares por día (divisiones/día) a partir de la siguiente fórmula:

$$
\mathrm{k}=3.332 \frac{\log N n-\log N o}{t n}
$$

donde $N n$ es la densidad celular al final del bioensayo; No es la densidad celular inicial nominal y $t n$ es el tiempo transcurrido entre el inicio y final del bioensayo (en días).

También se determinó el porcentaje de inhibición de la tasa de crecimiento (Ik) usando la siguiente fórmula:

$$
\mathrm{lk}=\frac{k c-k i}{k c} \times 100
$$

donde $k c$ es la tasa de crecimiento para la concentración $k$; y $k i$ corresponde a la tasa de crecimiento promedio para el control. El experimento se realizó por triplicado con muestreos diarios. 


\section{Análisis de pigmentos fotosintéticos}

Las muestras de células de E. gracilis fueron colectadas por centrifugación ( 2500 rpm) a las 72 y 144 h después de la exposición a las diferentes dosis de $\mathrm{Cu}^{2+}$. Posteriormente, $100 \mathrm{mg}$ de biomasa fresca de E. gracilis fueron homogenizados en oscuridad con $1 \mathrm{~mL}$ de acetona fría (100\%) por $1 \mathrm{~min}$. Una vez homogenizados, los tubos eppendorf de $2 \mathrm{~mL}$ conteniendo las muestras de E. gracilis y acetona fueron centrifugados a $2500 \mathrm{rpm}$ por $5 \mathrm{~min}$ a $4{ }^{\circ} \mathrm{C}$. Finalizado el proceso de centrifugación, el sobrenadante de cada muestra fue colectado y el contenido de carotenoides totales y clorofila a y $\mathrm{b}$ fueron evaluados a 470 y 662, $649 \mathrm{~nm}$ usando un espectrofotómetro. Donde se empleo las ecuaciones de Lichtentaler y Wellbum, ${ }^{6}$ que a continuación se describen para el cálculo de los pigmentos fotosintéticos:

$$
\begin{gathered}
\mathrm{Ca}=11.75 \mathrm{~A} 662-2.350 \mathrm{~A} 645 \\
\mathrm{Cb}=18.61 \mathrm{~A} 645-3.960 \mathrm{~A} 662 \\
\mathrm{Cx}+\mathrm{c}=1000 \mathrm{~A} 470-2.270 \mathrm{Ca}-81.4 \mathrm{Cb} / 227
\end{gathered}
$$

Donde $\mathrm{Ca}$ (clorofila a); $\mathrm{Cb}$ (clorofila b); y $\mathrm{C}_{\mathrm{X}+\mathrm{C}}$ (carotenoides totales)

\section{Análisis de la fragmentación del ADN de Euglena gracilis}

Biomasa de E.gracilis (100 mg) expuestas a las diferentes dosis del metal fueron colectadas por triplicado al final del experimento de acuerdo a la técnica propuesta por González-Mendoza et al.. ${ }^{7}$ Una vez obtenido las muestras de ADN, estas fueron observadas por electroforesis en gel de agarosa (0.8\%) teñido con bromuro de etidio por $25 \mathrm{~min}$. Posteriormente los fragmentos fueron visualizados y el porcentaje de daño se calculó por densitometria usando el programa image j.

\section{Análisis estadístico}

Los datos fueron analizados mediante el programa estadístico STATISTICA (Statistical Package version 5.5, Statsoft, USA) mediante una prueba de ANOVA y prueba de comparación de medias de Tukey $(\alpha=0.05)$.

\section{RESULTADOS Y DISCUSIÓN}

Entre los elementos potencialmente tóxicos (EPTs) de interés ecotoxicológico, el cobre tiene gran relevancia por sus efectos fisiológicos en los organismos ya que alteran diversas enzimas esenciales en los procesos metabólicos celulares. En el presente trabajo el crecimiento de E. gracilis disminuyó al aumentar las dosis del metal (Figura 1), siendo significativamente mayores la inhibición del crecimiento $(0.54$ y 0.30 ) con las dosis de 0.8 y $1.6 \mathrm{mM}$ de $\mathrm{Cu}$, respectivamente, al final del experimento (Figura 2). Por otra parte al evaluar el porcentaje de inhibición de la tasa de crecimiento se observó que la dosis media inhibitoria $\left(\mathrm{IC}_{50}\right)$ fue de $0.66 \mathrm{mM}$ de $\mathrm{Cu}$ al final del experimento. En este sentido, nuestros resultados contrastan con lo reportado por Einecker et al., ${ }^{8}$ quien ęncontró que la dosis media inhibitoria media fue de $0.22 \mathrm{mM}$ de $\mathrm{Cu}$.

Así mismo, Rochetta y Kupper ${ }^{9}$ encontraron que dosis de 50 $\mu \mathrm{M}$ de $\mathrm{Cu}^{2+}$ causaron el $90 \%$ de muerte en células de E. gracilis a los 7 días de exposición. Aunque hay que considerar que para tener una comparación confiable entre los diferentes experimentos que se han realizado y nuestros resultados, se requiere que factores como la concentración del metal y condiciones de cultivo del organismo empleados en las diferentes investigaciones sean similares. Otro parámetro empleado para valorar la capacidad de adaptación de $E$.

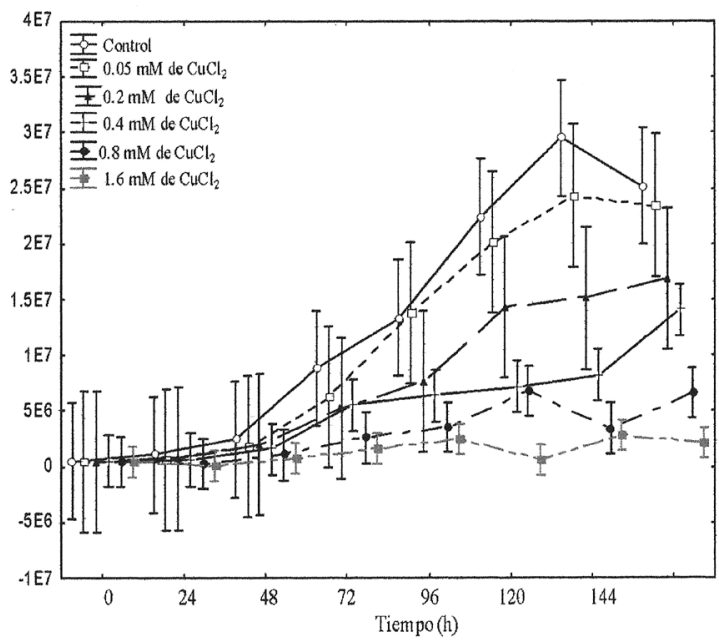

Figura 1. Curva de crecimiento de Euglena gracilis expuestas a diferentes dosis (mM) de $\mathrm{CuCl}_{2}$ a las 0, 24, 48, 72, 96,120 y $144 \mathrm{~h}$ después de la exposición al metal

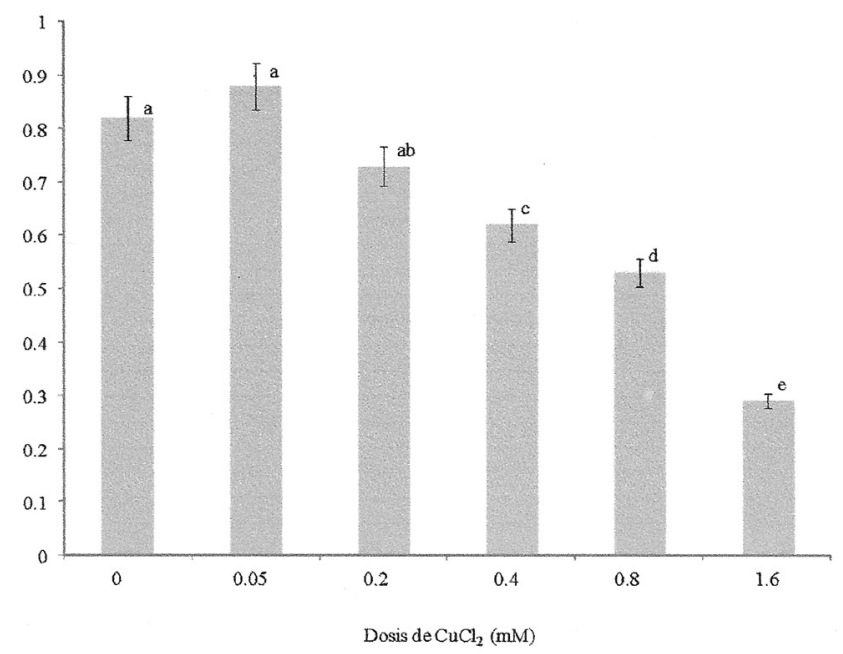

Figura 2. Efecto de diferentes dosis de $\mathrm{CuCl}_{2}(0,0.05,0.2,0.4,0.6,0.8$ y 1.6 $\mathrm{mM}$ ) en la tasa de crecimiento $(k)$ de células de Euglena gracilis después de 144 h de exposición. Barras de error estándar con letras diferentes son estadísticamente significativas ( $p \leq 0.05$ )

gracilis al $\mathrm{Cu}^{2+}$ fue la producción de pigmentos fotosintéticos, los cuales están relacionados con la viabilidad celular ya que se asocian con la capacidad fotosintética del organismos. ${ }^{10}$ En este sentido, los resultados mostraron que la producción de clorofila $a, b$ y carotenoides totales presentaron un comportamiento dosis dependiente ya que se observó una disminución significativa a mayores dosis del metal (Figura 3). Esta reducción en las concentraciones de pigmentos fotosintéticos pueden ser el resultado del efecto directo de los iones de $\mathrm{Cu}^{2+}$ que pueden inhibir la síntesis de la clorofila y carotenoides. ${ }^{11}$ El posible modo de acción del metal en los pigmentos fotosintéticos podría ser debido al incremento de especies reactivas de oxigeno (vía reacción de Fenton), y la disminución de la capacidad antioxidante de la célula. Así como a la capacidad de los iones de $\mathrm{Cu}$ de interactuar con los grupos $\mathrm{N}$ y $\mathrm{S}$ de proteínas y alterar la permeabilidad de la membrana. ${ }^{12}$ Similares resultados han sido observados en Scenedesmus incrassatulus (Chlorophyceae) donde dosis concentraciones subletales de $\mathrm{Cu}$, ocasionaron una rápida inactivación del fotosistema II, posiblemente como resultado de una disminución de los pigmentos fotosintéticos. ${ }^{13}$ 




Figura 3. Cambios en los niveles de pigmentos fotosintéticos de Euglena gracilis expuestas a 0, 0.05, 0.2, 0.4, 0.6, 0.8 y $1.6 \mathrm{mM}$ de $\mathrm{CuCl}_{2}$ después de 144 h de exposición. Barras de error estándar con letras diferentes son estadísticamente significativas ( $p \leq 0.05$ )

Por otra parte, los radicales libres generados por la exposición a metales pueden alterar el balance redox de las bases pirimidínicas y dañar al ADN. ${ }^{14}$

En este sentido nuestros resultados mostraron que la exposición de E. gracilis a las dosis altas de $\mathrm{Cu}^{2+}(0.8$ y $1.6 \mathrm{mM})$ generaron cambios significativos en la integridad del ADN (\% de daño del ADN) comparado con el control (Figura 4).

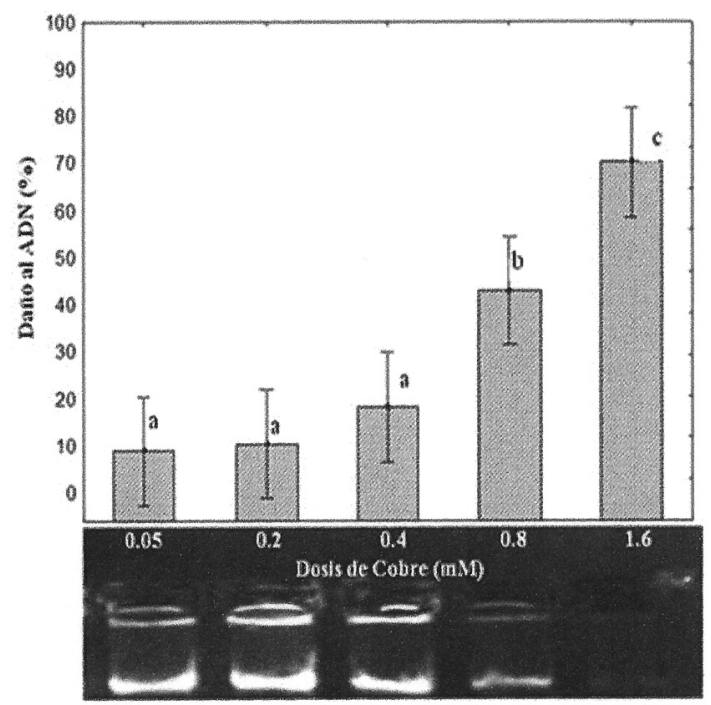

Figura 4. Niveles de daño del ADN en Euglena gracilis tratada con 0.05, 0.2, 0.4, 0.8 y $1.6 \mathrm{mM}$ de $\mathrm{CuCl}_{2}$ a las 144 h después de la exposición. Barras de error estándar con letras diferentes son estadísticamente significativas $(p \leq 0.05)$

Esto posiblemente a que el $\mathrm{Cu}^{2+}$ ha mostrado tener un mayor impacto en la modificación de las bases nitrogenadas del DNA, reflejándose en un mayor daño celular. ${ }^{14}$ Estos resultados son similares con lo reportado por Cai y Cherian ${ }^{15}$ en donde la exposición de células a metales a diferentes dosis generaba daños visibles en la integridad del ADN, indicando su posible función como un biomarcador de efecto a la exposición de metales. Por otra parte, se observó que los diversos efectos observados en los pigmentos fotosintéticos y en la integridad del ADN se incrementaron de manera proporcional a la cantidad de metal presente en el medio de cultivo. Estos resultados, desde el punto de vista ecotoxicológico, tienen relevancia para emplear a Euglena gracilis como un organismo bioindicador de contaminación por elementos potencialmente tóxicos (eg. cobre) en sistemas acuáticos. Lo anterior también ha sido propuesto por Ahmed y Häder ${ }^{16}$ quienes observaron que dosis crónicas de cobre tienden a generar cambios en parámetros fotosintéticos (eg. fluorescencia de la clorofila $a$ ) en $E$. gracilis. No obstante, el uso de los pigmentos fotosintéticos (clorofila y carotenoides) y degradación de DNA podrían ser herramientas que podrían contribuir a una mayor aceptación del uso de este organismo como agente indicador de contaminación del agua por metales esenciales. Por otra parte la presencia de moléculas como fitoquelatinas y compuestos fenolicos, pueden actuar en los procesos de acumulación de metales como cadmio en células de E. gracilis. $^{4}$

Por lo que es muy posible que la participación de mecanismos como, la producción de ácidos orgánicos, producción de polisacáridos, y/o la presencia de ligandos de alta afinidad, contribuyan al proceso de acumulación de $\mathrm{Cu}^{2+}$ por la biomasa de E. gracilis (variable no evaluada). Por lo que futuros estudios deben ser encaminados para el estudio de la capacidad de bioacumulacion de cobre y de los posibles mecanismos que participarían. Lo cual contribuirá a desarrollar estrategias para el uso de este organismo en procesos de biorremediación en ecosistemas acuáticos.

\section{CONCLUSIONES}

En el presente estudio el uso de los pigmentos fotosintéticos y de daño en la integridad del ADN, evaluados pueden ser empleados como indicadores fisiológicos para entender en parte el modo de acción del cobre en el aparato fotosintético del E. gracilis. Por otra parte, la capacidad de tolerancia de $E$. gracilis $(0.66 \mathrm{mM})$ nos indica la posible presencia de mecanismos de tolerancia a este metal. Así como el uso de E. gracilis como una herramienta potencial en los procesos de diagnostico de salud de ecosistemas acuáticos expuestos a cobre.

\section{AGRADECIMIENTOS}

Al Consejo Nacional de Ciencia y Tecnología (CONACyT) por el financiamiento de esta investigación mediante el proyecto No 79234. Así como al Programa de Mejoramiento del Profesorado (PROMEP).

\section{REFERENCIAS}

1. Gale, S. A.; King, C. K.; Hyne, R. V.; Environ. Toxicol. Chem. 2006, 25, 1887.

2. Yruela, I.; Braz. J. Plant Physiol. 2005, 17, 145.

3. Mullapudi, S.; Siletzky, M.; Kathariou, S.; Appl. Environ. Microbiol. 2008, 75, 1464.

4. Rodríguez-Zavala, J. S.; García-García, J. D.; Ortiz-Cruz, M. A.; Moreno-Sánchez, R.; J. Environ. Sci. Health, Part A: Toxic/Hazard. Subst. 2007, 42, 1365.

5. Mendoza-Cózatl, D. G.; Moreno-Sánchez, R.; Biochim. Biophys. Acta 2005, 1706, 88.

6. Lichtenthaler, H. K.; Wellburn, A. R.; Biol. Soc. Trans. 1985, 11, 591.

7. González-Mendoza, D.; Argumedo-Delira, R.; Morales-Trejo, A.; Pulido-Herrera, A.; Cervantes-Díaz, L.; Grimaldo-Juarez, O.; Alarcón, A.; Genet. Mol. Res. 2010, 9, 162.

8. Einicker-Lamas, M.; Mezian, G. A.; Fernandes, T. B.; Silva, F. L.; Guerra, F.; Miranda, K.; Attias, M.; Oliveira, M. M.; Environ. Pollut. 2002, 120, 779 .

9. Rocchetta, I.; Küpper, H.; New Phytol. 2009, 182, 405. 
10. MacFarlane, G. R.; Burchett, M. D.; Mar. Pollut. Bull. 2001, 42, 233.

11. Xing, W.; Huang, W. M.; Liu, G. H.; Environ. Toxicol. 2010, 25, 103.

12. González-Mendoza, D.; Quiroz-Moreno, A.; Medrano, R. E.; GrimaldoJuarez, O.; Zapata-Perez, O.; Z. Naturforsch., C: J. Biosci. 2009, 64, 391.

13. Perales-Vela, H. V.; Gonzalez-Moreno, S.; Montes-Horcasitas, C.; Canizares-Villanueva, R. O.; Chemosphere 2007, 67, 2274.
14. Frenzilli, G.; Ferrucci, M.; Giorgi, F. S.; Blandini, F.; Nigro, M.; Ruggieri, S.; Murri, L.; Paparelli, A.; Fornai, F.; Behav. Pharmacol. 2007, 18, 471.

15. Cai, L.; Cherian, M. G.; Toxicol. Lett. 2003, 136, 193.

16. Hoda, A.; Donat-Peter, H.; J. Appl. Phycol. 2010, 22, 785. 